\title{
SISTEMA PARA EL CÁLCULO DE REDES DE ACUEDUCTO
}

\author{
Autores \\ Jesús R. Hechavarría Hernández \\ Alexis Cordovés García. \\ Manuel García Pérez. \\ Centro de Estudios CAD/CAM \\ Universidad de Holguín - Cuba.
}

\section{RESUMEN}

El diseño de sistemas de suministro de agua cuenta en la actualidad con programas que han reducido notablemente la fuerza de trabajo y el tiempo necesario para realizarlo; no obstante, continúa dependiendo de información procedente de diferentes áreas o especialidades, así como del uso de dibujantes para obtener los planos ejecutivos.

El presente trabajo está concebido para resolver todos los problemas concernientes a los cáculos hidráulicos, diseño de la red y dibujo de los planos ejecutivos, también incluye el cálculo de los volúmenes de trabajo y listado de componentes industriales.

El método concebido por Hardy Cross para estabilizar hidráulicamente la red, ha sido totalmente automatizado incluyendo la distribución inicial de los caudales por los tramos y el sentido del flujo.

Se brindan opciones que facilitan la confección de la red, como son: el trazado de las tuberías, la ubicación de los nudos y tanques, entre otras. La ilustración gráfica de los resultados permite que se pueda conocer de forma rápida y precisa lo que acontece en el sistema. La información almacenada en las entidades: manzanas, nudos, tramos de tube-ría, tanques y otros componentes del sistema, pueden ser visualizadas en pantalla mediante diálogos de ac-tualización.

La metodología implementada en el sistema permite la reducción de las fuentes de información y consecuente disminución de errores concernientes a la manipulación de datos además de integrar toda la información del proyecto en soporte magnético lo que permite elevar la productividad de las empresas.

\section{INTRODUCCIÓN}

El uso de software destinados a la solución particularizada según las necesidades de diseño en sectores específicos de la ingeniería es una tendencia en la producción contemporánea, donde la reducción de los plazos de entrega del producto y el incremento constante de la calidad constituyen premisas para mantener los niveles de competitividad requeridos. Esta realidad ha conllevado a que diversos grupos de investiga-ción realicen trabajos encaminados a obtener sistemas computacionales con una elevada aplicación.

De esta forma surge como problema científico de esta investigación, la carencia de un software que permita diseñar una red de acueducto, tomando como referencia el dibujo de la ciudad en soporte magnético compatible con AutoCAD.

El objeto de este trabajo está enmarcado en el estudio y análisis de referencias bibliográficas y programas computacionales que abor- den la temática del dibujo y cálculo de redes de acueductos, teniendo como objetivo principal, la confección de un software que permita diseñar una red de acueducto, donde los proyectistas puedan concentrarse en los aspectos técnicos y en la toma de decisiones respecto a variantes concebidas en el programa.

Para dar solución al objetivo del tra-bajo se acometieron las siguientes tareas.

- Revisión bibliográfica a fin de conocer lo que, a este respecto, existe.

- Revisar hasta qué punto el objetivo del trabajo ha sido considerado en los software existentes.

- Repaso de métodos de iteración para resolver ecuaciones de la for-ma $f(x)=0$.

- Repaso de las ecuaciones funda-mentales de la hidráulica.

- Estudio de los métodos de cálcu-lo para las redes cerradas.

- Estudio del método de HardyCross para el cálculo de redes de acueducto.

- Análisis de las metodologías a se-guir para el diseño de redes de acueductos.

Los programas disponibles actualmente para proyectar redes de acueducto han mejorado las condicio-nes de trabajo, pero carecen aún de ilustraciones gráficas sobre el comportamiento de la red; se basan además, en la entrada de información que se almacena en forma matricial, requiriéndose en muchos casos, cálculos previos antes de introducir los datos. 
La novedad del trabajo consiste en la posibilidad de confeccionar y calcular una red de acueducto, tomando como base toda la información gráfica necesaria en formato compatible con AutoCAD, lo que incluye además los siguientes aportes:

- Disminuye los errores al integrar toda la información involucrada en cada elemento de la red.

- El uso de la ecuación de Darcy \& Weisbach para el cálculo de las pér-didas de energía garantiza diseños más exactos.

- La posibilidad de clacular los diferentes elementos de la red introduciendo modificaciones tanto de diseño como en sus componentes, facilita el análisis de variantes.

- Brinda facilidades para las labores de mantenimiento y actualización de diseños existentes.

- La utilización de software en el diseño de acueductos permite la reducción de los plazos de entrega y el incremento de la calidad de los proyectos, requisitos indispensables en las empresas proyectistas para mantener los niveles de competitividad requeridos.

- Desde el punto de vista social, el sistema mejora las condiciones de trabajo de los diseñadores ya que éstos podrán concentrarse en los as-pectos técnicos sin mayor atención al trazado, dibujo y cálculo.

- Contar con un software de aplicación sobre AutoCAD 14 que per- mita mostrar de forma rápida y pre-cisa los resultados del cálculo según las condiciones asignadas a una red cerrada de acueducto.

- La metodología aplicada para el cálculo podrá ser utilizada en la enseñanza de esta temática en asignaturas afines de carreras de Ingeniería.

- Por la información almacenada en las manzanas se pueden obtener representaciones sobre densidades reales y futuras, favoreciendo la toma de decisiones y la posible ubicación de los tanques de compensación.

Actualidad de programas que realizan el cálculo de redes de acueducto

El estudio de varios programs que realizan el cálculo de redes de acue-ducto nos ha llevado a la conclu-sión de que antes de comenzar a utilizarlos se deben realizar las si-guientes actividades:

1. Contar con los planos que mues-tren toda la información topográfica necesaria para realizar el diseño de la red.

2. Determinar la población y densidades futuras de cada manzana perteneciente al perímetro urbano. Durante este proceso se deben calcular las áreas de las manzanas a partir de la descomposición en figuras conocidas, por lo que es un proceso agotador tras realizar una cantidad importante de mediciones sobre el plano.

3. Realizar el trazado de todas las tuberías principales (se definen los circuitos y nudos).
4. Se define las áreas abastecidas y se calcula el caudal máximo hora-rio para cada nudo.

5. Se asume un sentido lógico de circulación del flujo en dependencia de la entrada del agua a la red y de acuerdo al gasto en tránsito entre un nudo y otro.

6. Se asume un coeficiente $\mathrm{C}$ (Hazen -Willian) según el diámetro y el tipo de tubería que se utilice.

7. Se calcula las longitudes de to-dos los tramos de tuberías, a par-tir de la información contenida en los planos.

8. Se codifica los elementos que intervienen en la red y se le asigna la información que le corresponda.

Durante el trasiego de información de los planos al papel y posteriormente a los programas disponibles es posible que se presenten errores que traerán como consecuencia resultados incorrectos.

Sistema DPReDSA para el diseño de redes de acueducto.

\section{Requisitos necesarios.}

Para la implementación del progra-ma, se necesita contar con los si-guientes requerimientos:

1.Hardware y software que permitan utilizar adecuadamente la versión 14 de AutoCAD.

2.Contar con la información topográfica necesaria para realizar el diseño de la red en soporte magnético (digitalización del plano). 


\section{Creación de las entidades que conforman la red.}

Sobre el dibujo en AutoCAD que representa el plano de la comunidad analizada, se crean los elementos fundamentales que intervienen en la red, como son: manzanas, niveles, nudos, tramos y tan ques. La manera de actualizar los datos referidos a las entidades se establece a tra-vés de diálogos como los que se muestran en los Anexos A y B.

\section{Creación de las Manzanas:}

El cálculo de la población futura es automatizado teniendo en cuenta las leyes urbanísticas establecidas para el municipio, donde se define, se-gún las hectáreas que ocupa cada manzana, las viviendas a construir y la cantidad de habitantes por vivienda que depende del estrato social a que pertenece la manzana. La población real se obtiene a partir de censos realizados a la comunidad. Poder acceder fácilmente a esta información permite comparar y corregir el cálculo de la población fu-tura la que depende de la pobla-ción actual de la manzana y la to-pografía del terreno.

Luego de establecer en un diálogo la información referente a población futura, población real, estrato social a que pertenece la manzana seleccionada, se muestra automáticamente, como resultados de cálculos, los valores de densidad futura, densidad real, caudal máximo horario y hectáreas que abarca la manzana.

El programa presenta opciones que permiten determinar y definir la re- lación que existe en el abastecimiento de las manzanas por los nudos.

\section{Creación de los Niveles:}

A partir de un punto seleccionado en el plano se introduce en un diálogo, la cota de nivel.

De esta manera se definen todas las cotas que sean de interés para el diseño; estás servirán de referencia para la ubicación de nudos, tanques y el trazado de los tramos. La cota piezométrica en el nivel será resul-tado del cálculo.

\section{Creación de los Nudos:}

A partir de un nivel seleccionado, se muestra en una caja de diálogo la cota de nivel. Los valores de cau-dal a consumir y población a abas-tecer, se determinan a partir de las manzanas que abastezca el nudo. La cota piezométrica en el nudo será resultado del cálculo.

\section{Creación de los Tramos:}

Mediante la opción de Crear tramos se dibuja una línea que parte de un Nudo Base siguiendo la trayectoria de los niveles que permiten los contornos de las calles y manzanas, hasta un segundo Nudo. De esta forma quedará determinada la longuitud de la tubería, la cual se muestra en la caja de diálogo referida a los tramos.

El tramo de tubería quedará definido luego de entrarle los datos referidos a material y diámetro. Los valores de caudal, pérdidas de presión y velocidad del fluido serán resultado del cálculo.

\section{Creación de los Tanques:}

Luego de seleccionar un nivel se di-buja en pantalla un símbolo que re-presenta la entidad tanque. Los va-lores de caudal y población a abas-tecer se determinan a partir de la de-manda en los nudos presentes en la red, los que "conocen " el caudal que necesitan para satisfacer los requeri-mientos de las manzanas que abas-tecen. La cota de nivel $(\mathrm{m})$ y la cota piezométrica $(\mathrm{m})$, así como la longuitud del tramo que comunica el tanque con la red y la ubicación exacta del símbolo tanque, serán resultado del cálculo cuando todos los nudos presenten presiones adecuadas.

Cálculo del caudal máximo horario para cada manzana.

Tomando como ejemplo una población de más de 60.000 habitantes el nivel de complejidad del sistema se clasifica como alto. Para un va-lor de 200L/hab-día como dotación neta y un porcentaje de pérdidas ad-misibles de un $20 \%$. La dotación bruta daría como resultado.

200 l/hab-día

dbruta $=\frac{}{1-0,2}$

dbruta $=250 \mathrm{l} /$ habdía

Para una manzana que presenta una población futura de 367 habitantes, el caudal medio será de:

367 hab. 250 l/hab- día Qmd

$$
86400
$$

$\mathrm{Qmd}=1,062 \mathrm{l} / \mathrm{s}$ 
Para un nivel de complejidad del sis-tema alto, $\mathrm{K}_{1}$ es igual a 1,20, dan-do como resultado un caudal máxi-mo diario:

$$
\begin{gathered}
\mathrm{QMD}=1,062 \mathrm{l} / \mathrm{s} \cdot 1,20 \\
\mathrm{QMD}=1,274 \mathrm{l} / \mathrm{s}
\end{gathered}
$$

Para un nivel de complejidad del sistema alto y una red matriz, $\mathrm{K}_{2}$ es igual a 1,40. El caudal máximo horario para la manzana analizada se define como:

$$
\begin{gathered}
\mathrm{QMH}=1,274 \mathrm{l} / \mathrm{s} \cdot 1,40 \\
\mathrm{QMH}=1,784 \mathrm{l} / \mathrm{s}
\end{gathered}
$$

De esta manera se determina automáticamente el caudal máximo horario de las manzanas a partir del valor de población futura que se halla definido para cada una.

\section{Cálculo del caudal máximo horario para cada nudo.}

Las opciones que brinda el programa donde se define la relación entre los nudos y las manzanas, ver figura $\mathrm{C} 1$ Anexo $\mathrm{C}$; permite que se pueda conocer el caudal máximo horario que necesita cada nudo, para satisfacer la demanda de agua de todas las manzanas abastecidas por él, a través del siguiente ecuación.



Donde:

QMHn: caudal máximo horario en el nudo
QMHm: caudal máximo horario en le manzana

m:número de manzanas abastecidas por el nudo

De esta manera se puede conocer el gasto total que debe abastecerse al sistema y las extracciones concentradas en los nudos.

\section{Determinación del caudal inicial para cada tramo}

Satisfaciendo la ley de continuidad de masa en los nudos, se realiza una distribución automática del caudal inicial por las tuberías, a partir de los tanques que intervienen, de manera que se define el sentido del flujo según la demanda de los nudos y el caudal que fluye por cada tramo, ver figura C.2, Anexo C. El sentido del flujo así como el caudal que trasiega por cada tubería serán corregidos durante el proceso de cálculo.

\section{Aplicación del método de Hardy Cross.}

A partir de la temperatura que presenta el agua en la región, las dimensiones, material y caudal inicial de cada tramo, se aplica el método de Hardy Cross. Como resultado del cálculo se obtendrá el valor del gasto, las pérdidas y la velocidad en cada una de las tuberías provocando la estabilidad hidráulica de la red.

La aplicación de la ley de conservación de la energía en los circuitos, la cual plantea que: "La suma algebraica de las pérdidas de energía en los tramos que conforman un anillo cerrado debe ser igual a cero", garantiza que cada circuito se comparte como un sistema de tuberías en paralelo. Posibilitando el cálculo de la presión en cada nudo de la red a partir de valores conocidos de presión en los nudos de abastecimiento, mediante la expresión establecida por Bernoulli.

\section{Opciones de visualización}

Durante el diseño de un sistema de acueducto, el especialista debe contar con cierta información gráfica que le ayude en la toma de decisiones durante el análisis, de variantes, como es el caso de la visualización del comportamiento de la densidad futura y real de las manzanas a través de una escala de colores. Esta información resulta ser interesante para valorar la formación de posibles sectores y ubicación de los tanques a proyectar. Ver anexos A y D.

El programa brinda las siguientes opciones de visualización:

a)Relación entre el abastecimiento de las manzanas por los nudos.

- Manzanas abastecidas por un nudo seleccionado.

- Manzanas abastecidas por los nudos.

- Nudos que abastece una manzana seleccionada

- Nudos que abastecen a las manzanas

b) A partir de una escala de colores que se refieren a valores de densidad de población se muestran las manzanas según: 
-Densidad real.

-Densidad futura.

c)Representación de las manzanas según el estrato social al que pertenecen.

d)Representación de los niveles según la cota nivel en metros.

\section{CONCLUSIONES}

De la investigación bibliográfica realizada, así como de las consultas con centros de proyecto se concluyó que no existen antecedentes de software ni aplicaciones sobre AutoCAD que ofrezcan los aportes que se reflejan en este trabajo.

Los programas existentes para el diseño de redes de acueductos carecen de herramientas gráficas que permitan visualizar los procesos, así como, de una adecuada interacción con el usuario en la toma de decisiones técnicas.

La utilización del AutoCAD como plataforma gráfica garantiza un ambiente de trabajo participativo, elimina gran cantidad de errores humanos y sustitiuye todo el trabajo de dibujo y cálculo en la elaboración de planos finales.

La aplicación del software a ejemplos de redes de acueductos calculadas en bibliografías, tales como la mostrada en la REVISTA AINSA, muestra una precisión en los cálculos hasta la milésima.

\section{BIBLIOGRAFÍA}

COLOMBIA. Reglamento Técnico para el sector de agua potable y saneamiento básico.1998. $550 \mathrm{p}$.

GERHART, Philip. R. y GROSS, J. Hochstein. Fundamentos de la Mecánica de los Fluidos. 2 ed. EUA: Addison Wesley, 1995. $1090 \mathrm{p}$.

GILES, Ronald. Mecánica de los fluidos. 2 ed. México: McGraw-Hill, 1967. 273 p.

KOUTITAS, Cristopher. Elements of Computational Hidraulics. London: Pentech ,1983.

KUTTZ, Myers. Enciclopedia de la Mecánica Ingeniería y Técnica. Vol. 6. España: Océano, 1995.

MARBELLO PÉREZ, Ramiro. Redes de distribución de agua. Medellín: s.n.,1990.

NEKRASSOV, B. Hidráulica. Moscú: Mir, 1990. 420 p.

PÉREZ FRANCO, Diosdado. Introducción al estudio de los sistemas de tuberías. La Habana: Pueblo y Educación, 1996. $120 \mathrm{p}$.

RABINOVICH, E. Hidráulica. Moscú: Mir, 1987. 316 p.

REVISTA AINSA: Publicación de la Asociación de Ingenieros Sanitarios y Ambientales. No.4 (ene.-jun. 1998); ISSN 0120-372X

STREETER, Victor. Mecánica de los fluidos. La Habana: Pueblo y Educación, 1980

SUÁREZ ALONSO, Margarita. Matemática Númerica, La Habana: Pueblo y Educación, 1980 


\section{ANEXO A}

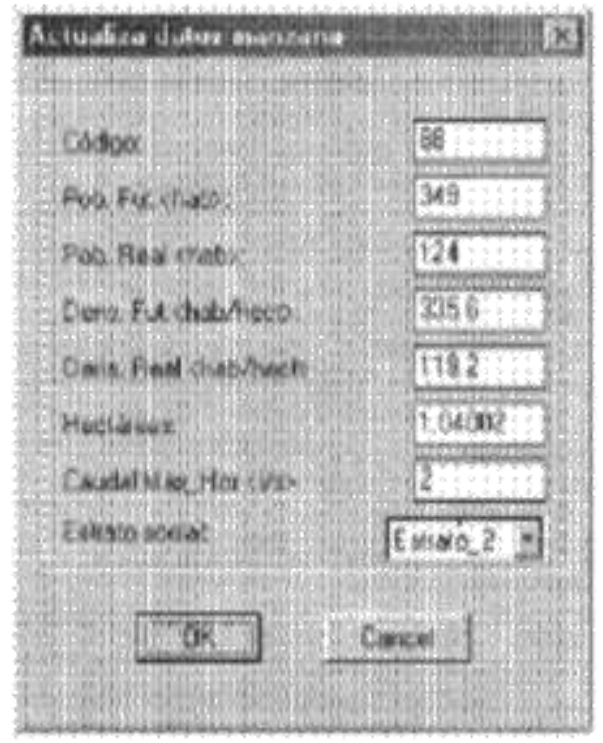

Figura A. 1 Diálogo que permite actualizar la información referida a la manzana seleccionada

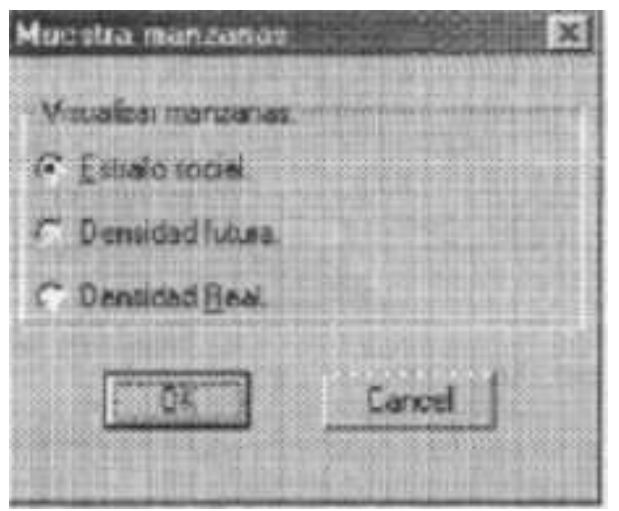

Figura A. 2 Diálogo que muestra las opciones de visualización de las manzanas según escala de colores 


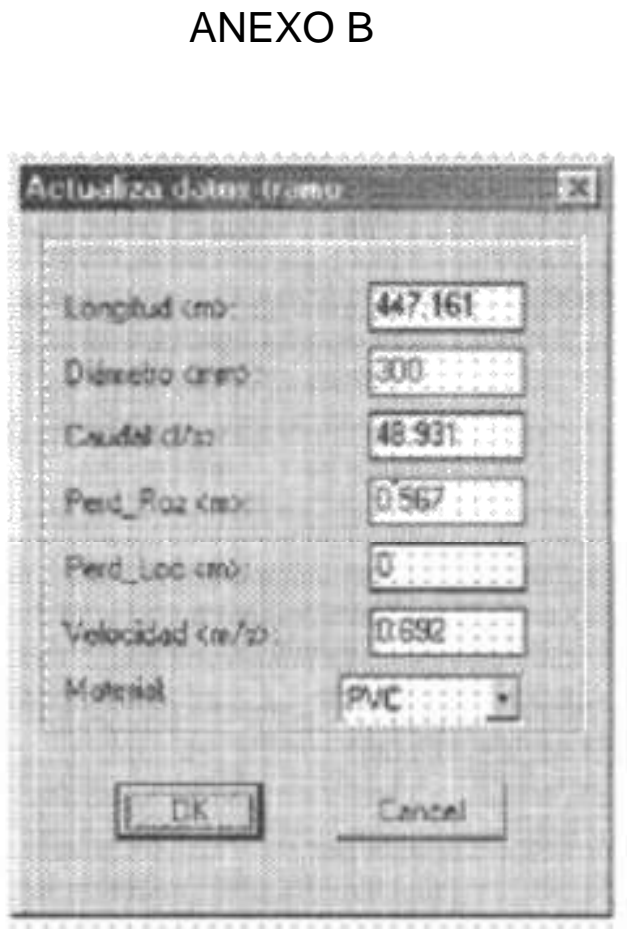

Figura B. 1 Diálogo que permite actualizar la información referida al tramo seleccionado

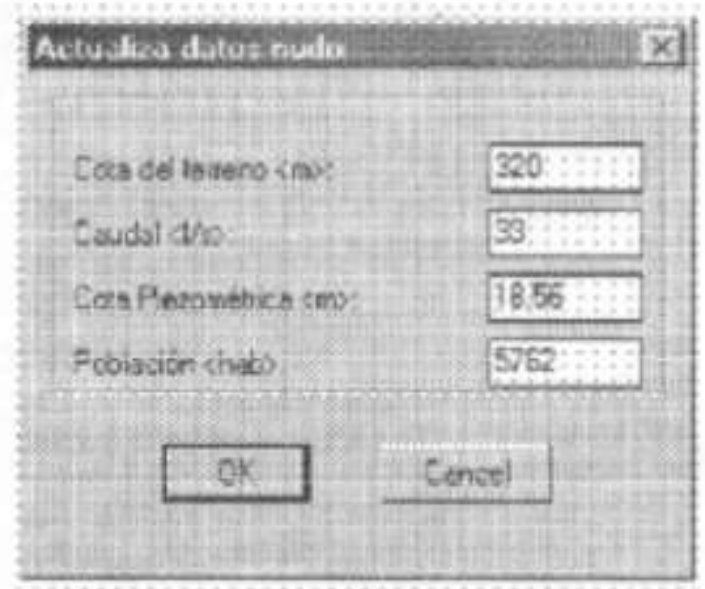

Figura B. 2 Diálogo que permite actualizar la información referida al nudo seleccionado 


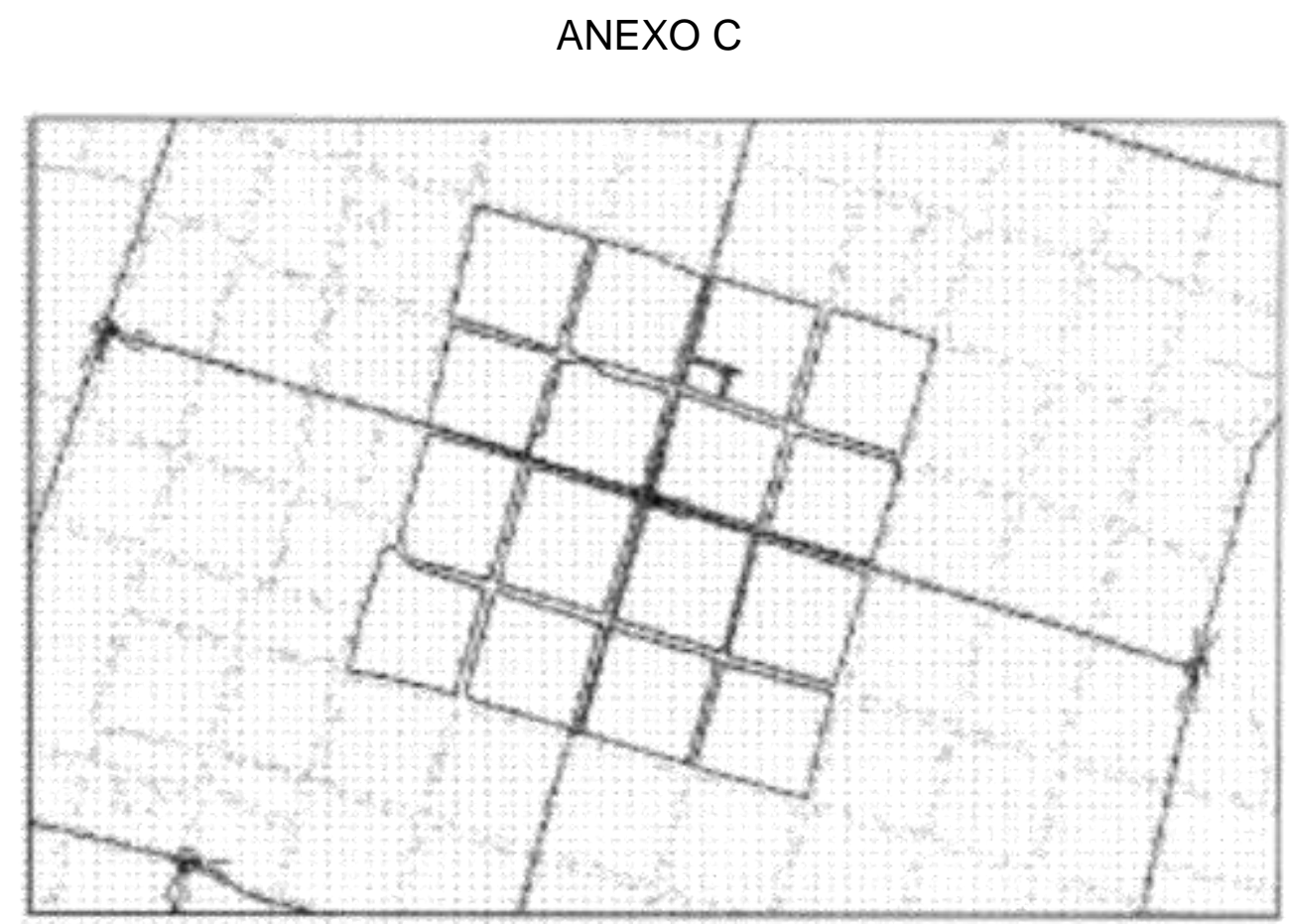

Figura C. 1 Esquema que muestra las manzanas abastecidas por el nudo seleccionado

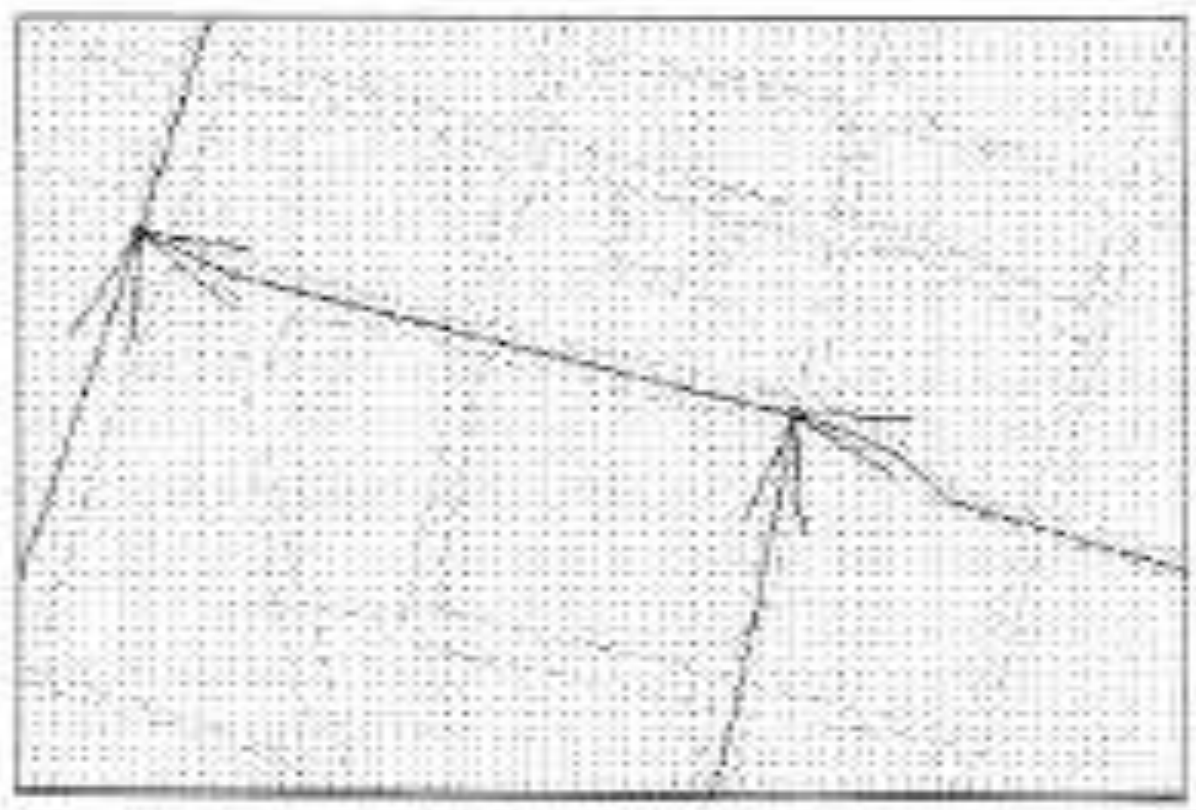

Figura C. 2 Esquema que muestra el sentido de circulación del flujo a través de las tuberías 


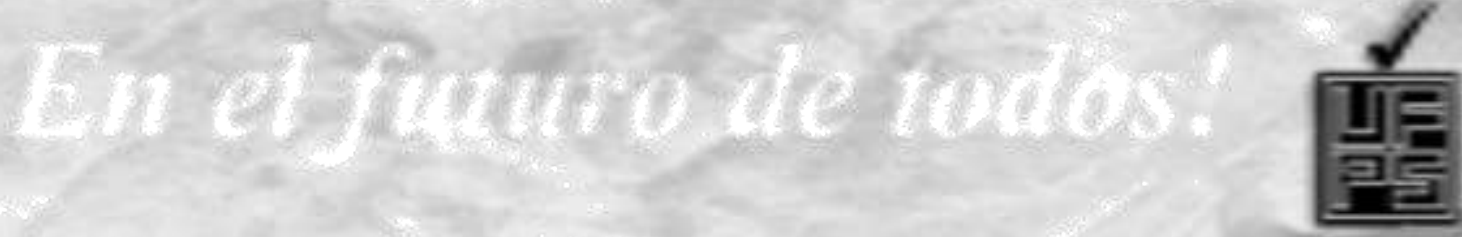

\section{ANEXO D}

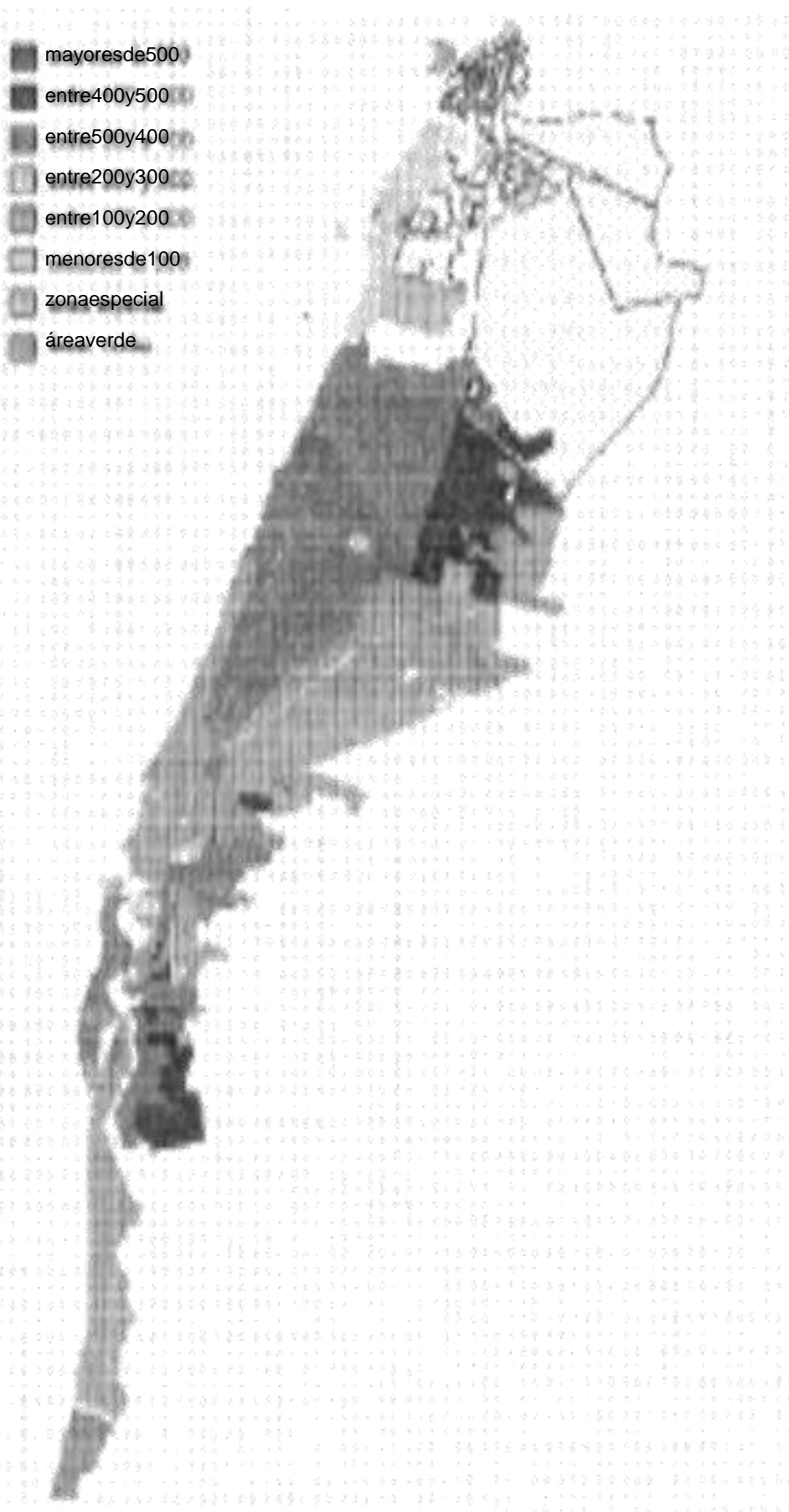

Figura D. 1 Esquema que muestra la presentación de las manzanas en una escala de colores según la densidad futura de una comunidad dada. 
